\title{
Red Supergiant Stars as Supernova Progenitors - the X-ray Perspective
}

\author{
Vikram V. Dwarkadas \\ Dept of Astronomy and Astrophysics, U Chicago, 5640 S Ellis Ave \\ email: vikram@oddjob.uchicago.edu
}

\begin{abstract}
Red Supergiants (RSGs) have for decades been assumed to be the progenitors of Type IIP supernovae (SNe). They are expected to have dense winds with mass-loss rates up to $10^{-4} \mathrm{M}_{\odot} \mathrm{yr}^{-1}$. We have created a database of available X-ray lightcurves of SNe. Type IIP $\mathrm{SNe}$ are found to have the lowest X-ray luminosities among all classes, which is surprising given the high mass-loss rate winds expected from their red supergiant progenitors, and therefore the high density medium into which Type IIP SNe are expected to expand into. We show that the low X-ray luminosity sets a limit on the mass-loss rate of the progenitor star which can collapse to become a RSG, which is about $10^{-5} \mathrm{M}_{\odot} \mathrm{yr}^{-1}$. This in turn can be used to set a limit on the initial mass of a RSG star which can become a Type IIP progenitor, which is about $19 \mathrm{M}_{\odot}$. This is consistent with the limit obtained via direct optical progenitor identification. Optically identified progenitors of Type IIP SNe are found to be RSGs with masses less than about $17 \mathrm{M}_{\odot}$ (Smartt (2009)). We discuss the implications of this result for stellar evolution, theorize on the fate of RSG stars with initial mass $>19 \mathrm{M}_{\odot}$, and discuss what type of SNe they will produce at the end of their lifetime.
\end{abstract}

Keywords. shock waves, stars: mass loss, supernovae: general, stars: winds, outflows, X-rays: general, X-rays: ISM

\section{Introduction}

Type IIP SNe arise from red supergiant (RSG) stars. These stars have winds with mass-loss rates $\dot{M}$ ranging from $10^{-7}-10^{-4} \mathrm{M}_{\odot} \mathrm{yr}^{-1}$, and wind velocities $v_{w}$ from $10-50 \mathrm{~km} \mathrm{~s}^{-1}$ (Mauron \& Josselin (2011)). Thus they are expected to have high wind densities $\left(\propto \dot{M} / v_{w}\right)$ surrounding the star. Since thermal X-ray emission is proportional to the square of the ambient density, the X-ray lightcurve should reflect the radial structure of the ambient density profile if the emission is thermal. Figure 1 shows the observed X-ray lightcurves of several SNe. The X-ray luminosities of Type IIP SNe are found to be the lowest of all types. The low level of thermal emission is surprising given that the wind density should be high. The maximum observed X-ray luminosity of a Type IIP SNe is seen to be around $3 \times 10^{39} \mathrm{erg} \mathrm{s}^{-1}$. Using Chevalier \& Fransson (2003), this gives a maximum mass-loss rate of about $10^{-5} \mathrm{M}_{\odot} \mathrm{yr}^{-1}$ (Dwarkadas (2014)) for a RSG that collapses to form a Type IIP. The mass-loss rate of RSG stars is proportional to their initial mass; this leads to a maximum mass of $\approx 19 M_{\odot}$ for the initial mass of a Type IIP SN (Mauron \& Josselin (2011)). If the observed emission is not thermal, the mass-loss rate and thus initial mass could be even lower. It is possible that the flux from $\mathrm{SNe}$ evolving in higher density winds may be absorbed; the ionization level, and effects of X-ray ionization must be assessed to determine this. A detailed study of this and other factors can be found in Dwarkadas (2014). The results suggest that RSGs above $\approx 19 M_{\odot}$ do not tend to explode as Type IIP SNe.

What is the final fate of RSGs with initial mass above $19 M_{\odot}$ ? Presumably they should give rise to some of the other $\mathrm{SNe}$ shown in the figure. We do not expect them to form 


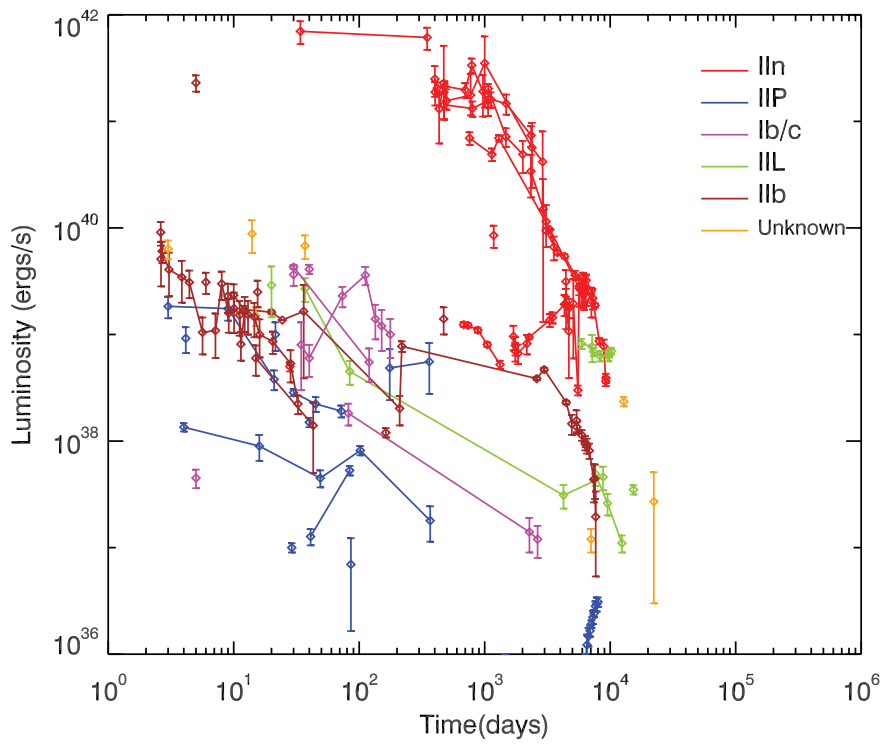

Figure 1. X-ray lightcurves of most observed SNe, grouped by type. Adapted from Dwarkadas \& Gruszko (2012), with more SNe and additional datapoints. It is clearly seen that the type IIP SNe have the lowest X-ray luminosities of all types, which is surprising given that they are expected to have a high density environment around them.

SNe of Type Ib/c, but it is possible that they could give rise to other types of SNe, such as IIL, IIb or even IIns. Dwarkadas (2011) had suggested that some IIns could arise from RSGs with a high mass-loss rate. Georgy et al. (2012) infer from their stellar evolution models that rotating stars above $16.8 M_{\odot}$, and non-rotating stars above $19 M_{\odot}$, do not collapse to form Type IIP SNe, but instead could form IIL or IIn SNe, consistent with the above results. Therefore, it is possible that RSGs could be the progenitors of several types of SNe.

\section{References}

Chevalier, R. A. \& Fransson, C. 2003, LNP, 598, 171

Dwarkadas, V. V. 2014, MNRAS, 440, 1917

Dwarkadas, V. V. \& Gruszko, J. 2012, MNRAS, 419, 1515

Dwarkadas, V. V. 2011, MNRAS, 412, 1639

Georgy, C., et al. 2012, A\&\&A, 542, A29

Mauron, N. \& Josselin, E. 2011, A\& $A$, 526, 156

Smartt, S. J. 2009, ARAA, 47, 63 